\title{
FLASHES AND FLOATERS AS PREDICTORS OF VITREORETINAL PATHOLOGY: IS FOLLOW-UP NECESSARY FOR POSTERIOR VITREOUS DETACHMENT?
}

\author{
M. R. DAYAN, D. G. R. JAYAMANNE, R. M. ANDREWS and P. G. GRIFFITHS \\ Newcastle upon Tyne
}

\begin{abstract}
SUMMARY
Purpose. The aim of the study was to determine whether patients presenting with an isolated posterior vitreous detachment require follow-up to identify retinal breaks not apparent at presentation and whether some histories are more predictive of associated serious posterior segment pathology.

Methods. The notes of 295 patients presenting to eye casualty with flashes and/or floaters were reviewed.

Results. One hundred and eighty-nine patients (64\%) had isolated posterior vitreous detachments, 49 $(16.6 \%)$ had retinal detachments and $31(10.5 \%)$ had flat retinal tears. Three new breaks $(3.3 \%$ of all tears found, $1.9 \%$ of review appointments) were identified only at follow-up. Although a subjective reduction in vision and a history of less than 6 weeks' duration were strongly predictive of retinal breaks, the large group of patients presenting with floaters alone $(124 / 295,42 \%)$ still harboured a significant proportion $(26.7 \%)$ of the retinal breaks.

Conclusions. A follow-up visit for patients with an isolated posterior vitreous detachment can be justified to detect the small percentage of asymptomatic retinal breaks. Although a subjective reduction of vision is the symptom most predictive of serious posterior segment pathology, it would be unsafe to identify particular subgroups of patients alone for careful examination.
\end{abstract}

Patients complaining of flashes and floaters make up a significant proportion of the cases presenting to eye casualty departments. Of these, between $10 \%$ and $30 \%{ }^{1-3}$ will have retinal breaks requiring immediate treatment and approximately half will have an isolated posterior vitreous detachment. ${ }^{1,4}$ The latter

From: Department of Ophthalmology, Newcastle General Hospital, Newcastle upon Tyne, UK.

Correspondence to: Miss Margaret R. Dayan, Department of Ophthalmology, Royal Victoria Infirmary, Queen Victoria Road, Newcastle upon Tyne NE1 4LP, UK. Fax: +44 1912275246. group are usually followed up in outpatients approximately 6 weeks after the onset of symptoms, to allow the identification of retinal breaks which may have developed after the first visit.

Our aims were to determine whether significant pathology associated with posterior vitreous detachment was being detected at the follow-up visit and to identify characteristics of the history which were more strongly indicative of the presence of retinal breaks or detachments.

\section{PATIENTS AND METHODS}

We retrospectively studied 295 consecutive patients who presented to eye casualty in 1993 and 1994 with symptoms of floaters and/or flashes due to posterior vitreous detachment. Patients who were referred directly to outpatients, or who had a history of trauma or concurrent posterior segment disease such as diabetic retinopathy and vascular occlusions, were excluded. All had been examined at the first visit by the senior house officer in casualty and a diagnosis of separation of the posterior vitreous face from the retina was made using slit lamp biomicroscopy with the 90 dioptre lens or Goldmann 3-mirror contact lens. Indirect ophthalmoscopy with scleral indentation was also performed to identify any peripheral retinal pathology and patients were then treated immediately as necessary or given a retinal detachment warning and followed up in clinic. Patients' symptoms, pathology, timing of follow-up and findings on follow-up were recorded.

\section{RESULTS}

One hundred and twenty-four (42\%) patients with pathology presented with floaters alone, $53(18 \%)$ with flashes, $60(20.3 \%)$ with floaters and flashes and $58(19.7 \%)$ with floaters and/or flashes with a subjective reduction in vision. The complaint of 
reduced vision did not always correspond to a poor visual acuity on testing on the Snellen chart.

One hundred and eighty-nine patients $(64 \%)$ were found to have a posterior vitreous detachment alone at presentation. Thirty-one $(10.5 \%)$ had flat retinal tears, $49(16.6 \%)$ had retinal detachments, $3(1 \%)$ had vitreous haemorrhage with visible tears and 18 $(6.1 \%)$ had dense vitreous haemorrhages preventing an adequate fundal view, of whom 2 patients were subsequently found to have flat retinal tears when the haemorrhage cleared. One patient was found to have an area of lattice associated with a small round hole and an operculum. Four patients (1.4\%) were found to have other ocular pathology to account for their symptoms.

Thirty-nine $(67 \%)$ of the patients with subjective visual loss had retinal tears or detachments, compared with $14(23 \%)$ of those with flashes and floaters, $10(18.9 \%)$ of those with flashes and 23 $(18.5 \%)$ of those with floaters alone. However, due to the large number of patients presenting with floaters alone and the much smaller numbers presenting with additional visual loss, $45.4 \%$ of the tears and detachments occurred in patients with subjective visual loss but over a quarter $(26.7 \%)$ occurred in patients with floaters alone, $16.3 \%$ in those with floaters and flashes and $11.6 \%$ in those with flashes. The length of history was also linked to the presence of serious pathology, with all but 1 $(98.8 \%)$ of the retinal breaks and detachments presenting within the first 6 weeks $(95 \%$ within the first 4 weeks) compared with only $95 \%$ of the isolated posterior detachments.

At presentation 84 patients $(28.4 \%)$ had retinal breaks or detachments of which 51 required surgery, 30 laser and 2 cryotherapy, with 1 patient with carcinoma of the lung being too ill to undergo retinal detachment repair. Forty-three patients $(14.6 \%)$ with posterior vitreous detachment alone and onset of symptoms more than 6 weeks previously were discharged from casualty at the first visit.

Fifty-five patients $(19.2 \%)$ returned to casualty prior to their scheduled follow-up appointment, but new pathology (a round hole requiring laser) was found in only 1 case. A total of 169 patients were given follow-up appointments of which 12 (7\%) failed to attend. Six retinal breaks were found at follow-up: 3 of these were suspected -2 were flat breaks visualised as vitreous haemorrhage cleared and 1 was a retinal hole which was provisionally identified at the initial assessment and confirmed at review the next day. However, one horsehoe tear, one operculated break and one round hole in an area of lattice were identified at follow-up by a consultant, a registrar and a different senior house officer $(3.3 \%$ of all the tears found, $1.9 \%$ of follow-up appointments) in patients who had suffered no further symptoms and had not returned prior to their appointment.

\section{DISCUSSION}

Eye casualty departments see many patients with flashes and floaters. Although up to a third of these patients have retinal breaks or detachments at presentation, over half will be identified as having an isolated posterior vitreous detachment and reviewed at 6 weeks after the onset of symptoms, as most symptomatic breaks which are going to detach will do so during this period.,

In our study we looked at 295 patients with posterior vitreous detachments who presented with flashes and floaters. Our results are comparable with those reported previously ${ }^{1-4}$ in that $188(63.7 \%)$ had isolated posterior vitreous detachments and 84 $(28.5 \%)$ had retinal breaks or detachments on presentation. Two patients $(0.7 \%)$ had breaks which were found later when their vitreous haemorrhage cleared and 1 had a hole which was suspected in casualty and confirmed at follow-up the next day. Fifty-five patients $(29.3 \%)$ with a diagnosis of posterior vitreous detachment returned to casualty with new symptoms prior to their follow-up appointment, of whom only 1 had new pathology - a flat round hole which was lasered.

Following up patients with posterior vitreous detachments involves considerable resources - in our department serving a catchment population of 1.1 million we gave follow-up appointments to 169 patients with posterior vitreous detachments during 1993 and 1994. However, of the 157 who attended their appointment, 3 were found to have breaks which had not been seen or suspected at their casualty visit: 1 with a horsehoe tear which definitely required treatment and 2 patients with a round hole in an area of lattice and an operculated hole who both received laser treatment, although the need for treatment in these cases is rather more controversial. $^{5-7}$ These three breaks therefore comprise 3.3\% of the total breaks and detachments identified, and occurred in $1.9 \%$ of the patients given follow-up appointments. It can be argued that only 1 of these patients (with a symptomatic horshoe tear) was at definite risk of retinal detachment and that the return in terms of pathology identified for the investment of clinic time in follow-up is very small. The decision of whether to follow up these patients is an individual judgement, but in our department we have chosen to continue the 6 week review of patients with isolated posterior vitreous detachment on the basis of the findings of this study.

The association between the presence of serious pathology and the type and duration of symptoms found in this study is similar to that reported in the literature, ${ }^{1,2,4,8,9}$ although in our study the presence 
of a subjective reduction in vision was found to be highly predictive of the presence of both retinal detachments and flat breaks - a link which has not been reported previously to the best of our knowledge. Such a reduction in vision without detachment could be due to the presence of small amounts of vitreous blood or pigment or to the breakdown of the blood-vitreous barrier with resultant vitreous flare. Thirty-nine patients (67\%) with flashes and/or floaters with a subjective reduction in vision had retinal breaks or detachments at presentation compared with 14 of $60(23 \%)$ with both flashes and floaters, 23 of $124(18.5 \%)$ with floaters alone and 10 of $53(11.6 \%)$ with flashes alone. However, it is worth noting that as $42 \%$ of all the patients presented with floaters alone, these patients accounted for over a quarter $(26.7 \%)$ of all the breaks. We did not find isolated photopsia to be any more predictive of serious pathology than floaters.

In keeping with previous studies, all but one of the breaks and detachments $(83 / 84,98.8 \%)$ presented to casualty with less than a 6 week history of symptoms, compared with only 179 of $188(95 \%)$ of the isolated posterior vitreous detachments - although our study was based on patients presenting to eye casualty to the exclusion of outpatient referrals, who tend to have a longer and less florid history of symptoms and who therefore may represent a different subgroup.

The yield of unsuspected, asymptomatic pathology detected at the 6 week follow-up of patients with isolated posterior vitreous detachments is low in this study $(1.9 \%$ of patients at follow-up), but the consequences of missed retinal breaks are potentially serious. In our department we have chosen to continue the 6 week review of these patients on the basis of these findings. Although those patients with a subjective reduction in vision are more likely to harbour retinal breaks, any complaint of flashes or floaters should prompt a very careful clinical examination at presentation.

Key words: Posterior vitreous detachment, Retinal detachment, Floaters, Photopsia.

\section{REFERENCES}

1. Diamond JP. When are simple flashes and floaters ocular emergencies? Eye 1992;6:102-4.

2. Boldrey EE. Risk of retinal tears in patients with vitreous floaters. Am J Ophthalmol 1983;96:783-7.

3. Jaffe NS. Complications of acute posterior vitreous detachment. Arch Ophthalmol 1968;79:568-71.

4. Hikichi T, Trempe CL. Relationship between floaters, light flashes, or both and complications of posterior vitreous detachment. Am J Ophthalmol 1994;117: 593-8.

5. Davis MD. Natural history of retinal breaks without detachment. Arch Ophthalmol 1974;92:183-94.

6. Neumann E, Hyams S. Conservative management of retinal breaks. Br J Ophthalmol 1972;56:482-6.

7. Combs JL. Retinal breaks without detachment: natural history, management and long term follow-up. Trans Am Ophthalmol Soc 1982;80:64-97.

8. Novak MA, Welch RB. Complications of acute symptomatic posterior vitreous detachment. Am J Ophthalmol 1984;97:308-14.

9. Brod RD, Lightman DA, Packer AJ, Saras HP. Correlation between vitreous pigment granules and retinal breaks in eyes with acute posterior vitreous detachment. Ophthalmology 1991;98:1366-9.

10. Kanski JJ. Complications of acute posterior vitreous detachment. Am J Ophthalmol 1972;80:44-6.

11. Murakami K, Jalkh AE, Avila MP, Trempe CL, Schepens CL. Vitreous floaters. Ophthalmology 1983; 90:1271-6.

12. Morse PH. Symptomatic floaters as a clue to vitreoretinal disease. Ann Ophthalmol 1975;7:865-8. 